\title{
Summary of Experimental Results: Photons, Leptons and Heavy Quarks
}

\author{
Richard K. Seto \\ Physics Department University of California, Riverside, Riverside, CA 92521, USA \\ E-mail: richard.seto@ucr.edu
}

\begin{abstract}
This is a summary of experimental results on photons, leptons, and heavy quarks presented at Quark Matter 2008. A first measurement of the bottom to charm contribution to the lepton spectrum has given experimental indication for the suppression of charm and bottom. Excess dileptons have been observed and studied by both NA60 and PHENIX, which may arise from the early production of thermal dileptons and/or the modification of mesons.
\end{abstract}

\section{Introduction}

The evidence for the production of a strongly interacting Quark Gluon Plasma (sQGP) at RHIC is very strong, however coming into this conference there are questions one would like to have answered. The hallmark signatures for the sQGP have been the suppression of high momentum particles, and the strong elliptic flow of hadrons. Surprisingly, heavy quarks seen via their leptonic decays have shown a similar behavior. Since both charm and bottom quarks decay leptonically, the first question one would like to answer is whether this behavior is limited to the charm quark, or includes the much heavier bottom quark as well. Secondly, since strong elliptic flow indicates that the system is thermalized, one is led to ask whether the "black body" radiation from this thermalized system in the form of thermal dileptons can be seen. Dileptons can pass through the system basically unhindered, hence they can give us information about the time evolution of the system - both from the aforementioned thermal radiation and from the decays of hadrons, or quasi-hadrons inside the high temperature region which may allow us to observe hadron masses and widths in a chirally symmetric phase. Finally we will ask whether new results in the ongoing story of the $\mathrm{J} / \psi$ can give us any indication of deconfinement.

\section{Heavy Quarks}

One of the most important early experimental results was the suppression of high momentum particles. The standard explanation for this effect is that high momentum quarks/gluons loose energy before exiting the medium. This signature is an indication of 
a very high energy density; estimates range from 25 to 100 times the density of nuclear matter. The strength of the elliptic flow leads one to believe that thermalization occurs very early, probably $\sim 1 \mathrm{fm} / \mathrm{c}[1]$ though some recent estimates have been much earlier than this. This leads to a picture of quarks, even quarks of relatively high momentum, being thermalized rather quickly (or nearly thermalized) because of the strength of the interaction they experience. This could come from a large coupling constant, or from a smaller coupling constant together with a turbulent system[2]. A striking, rather recent result, is that the interactions are so strong that heavy quarks such as charm also begin to thermalize and exhibit both suppression at high transverse momentum, and elliptic flow. Charm and bottom quarks, were not expected to thermalize and flow with the medium because of their mass $-\mathrm{m}_{c} \sim 1.3 \mathrm{GeV}, \mathrm{m}_{b} \sim 5 \mathrm{GeV}$. In addition, if the suppression of high $\mathrm{p}_{T}$ particles is a consequence of radiative energy loss, then the "dead-cone effect[3]" would mean that heavy quarks should show less suppression. Heavy quarks have been observed using non-photonic electrons (electrons spectra after the contribution of electrons from photon conversion and Dalitz decays are subtracted) which come from the semi-leptonic decay of both charm and bottom mesons. These indicate that heavy quarks are suppressed at high $\mathrm{p}_{T}$ [4] (see Fig. 1, left). An update of the non-photonic electron flow is shown in Fig. 1 (right) where the $\mathrm{v}_{2}$, now shown out to a $\mathrm{p}_{T}$ of $5 \mathrm{GeV}$, appears to saturate at about 0.07 .

Clearly charm flows and is suppressed at high $\mathrm{p}_{T}$ - what about bottom? Since heavy quarks are produced in the earliest moments of the collision via perturbative processes, their initial yield and $\mathrm{p}_{T}$ distribution in heavy ion collisions will be the same as in pp collisions scaled by the number of binary collisions. Both STAR and PHENIX have now made a measurement of the $\mathrm{b} / \mathrm{c}$ ratio in $\mathrm{p}+\mathrm{p}$ collisions. PHENIX uses a technique of examining the $\mathrm{K}+\mathrm{e}$ invariant mass distribution from the $\mathrm{D} \rightarrow K e \nu$ decay, subtracting off like sign combinations and fitting the result to a combination of $b$ and c contributions. STAR uses a similar method but in addition they directly reconstruct the $D \rightarrow K \pi$ decay with an electron tag (see Fig. 2, left). Fig. 2 (right) shows the $\mathrm{D} /(\mathrm{D}+\mathrm{B})$ ratio obtained from the two experiments; the results agree nicely with the FONLL expectations. The implication is that bottom is responsible for a substantial part of the non-photonic electron signal - about $50 \%$ at $\mathrm{p}_{T}$ greater than 3 or $4 \mathrm{GeV}$. Taken together with the $\mathrm{R}_{A A}$ and $\mathrm{v}_{2}$ results in $\mathrm{Au}+\mathrm{Au}$, this indicates that the $\mathrm{b}$ quark is at least partially thermalized in central $\mathrm{Au}+\mathrm{Au}$ collisions and accounts for a nonnegligible amount of the suppression and elliptic flow of non-photonic electrons. This is a very unexpected result since the relaxation time of the b-quark is much longer than that of the light quarks, because of its heavy mass. This would further imply that the strength of the interactions is strong - strong enough to force the bottom quarks to move with the bulk of the sQGP, hence non-perturbative calculations become necessary. A Langevin based model which includes resonant elastic scattering obtains a good fit to both $\mathrm{R}_{A A}$ and $\mathrm{v}_{2}$ with a diffusion coefficient $\mathrm{D}_{H Q}=(4$ to 6$) / 2 \pi \mathrm{T}[5]$.

One can then make an estimate for $\eta / \mathrm{s}$ using transport models via the diffusion coefficient for heavy quarks. Moore and Teaney calculate, using perturbative methods 
that $\mathrm{D}_{H Q} \sim 6 \eta /(\epsilon+\mathrm{P})[6]$ and give a plausibility argument that the result is true nonperturbatively. Since at $\mu_{B}=0, \epsilon+\mathrm{P}=\mathrm{Ts}$, one arrives at an answer for $\eta / \mathrm{s} \sim(1.3$ to 2.0) $/ 4 \pi[4$, close the the conjectured lower bound from AdS/CFT calculations[7] which assume extremely strong interactions.
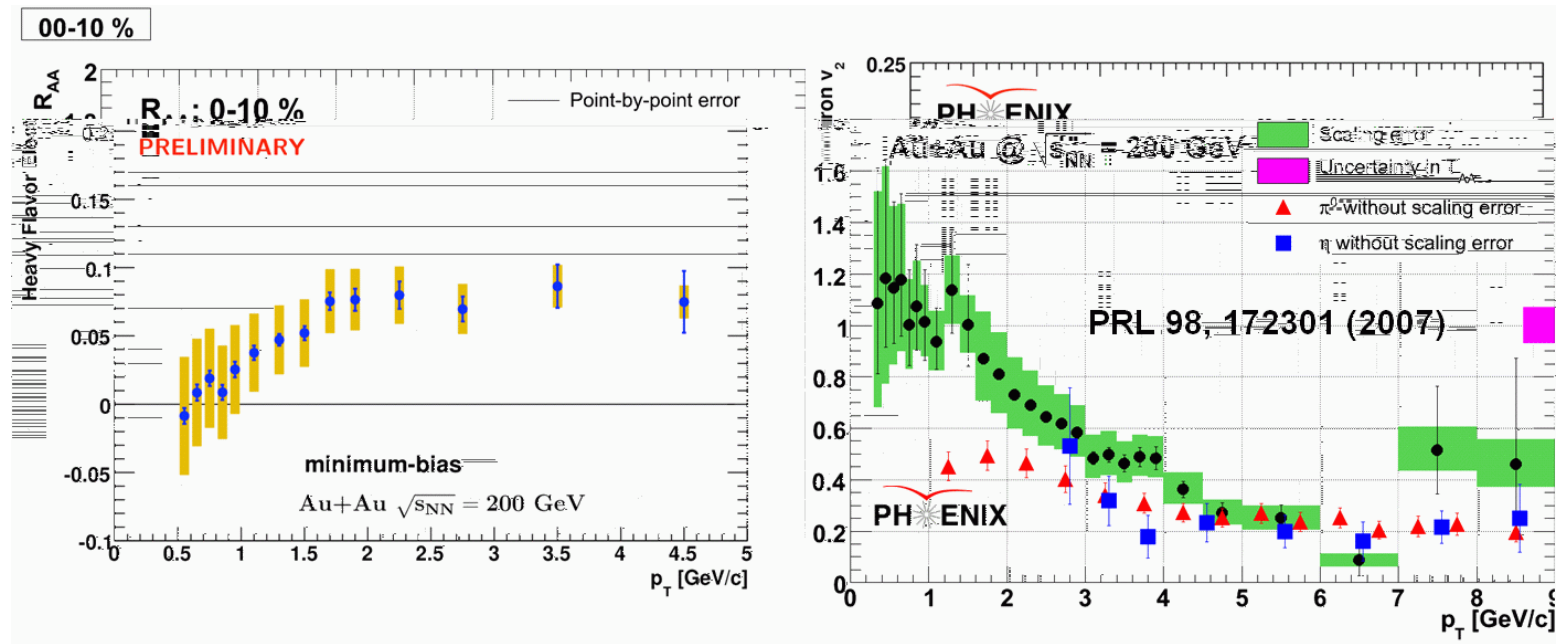

Figure 1. Left: $\mathrm{R}_{A A}$ for non-photonic electrons and $\pi^{0} \mathrm{~s}$ in central $\mathrm{Au}+\mathrm{Au}$ collisions. Right: $\mathrm{v}_{2}$ for non photonic electrons in minimum bias $\mathrm{Au}+\mathrm{Au}$ collisions.

\section{W/ non-photonic electron trigger}

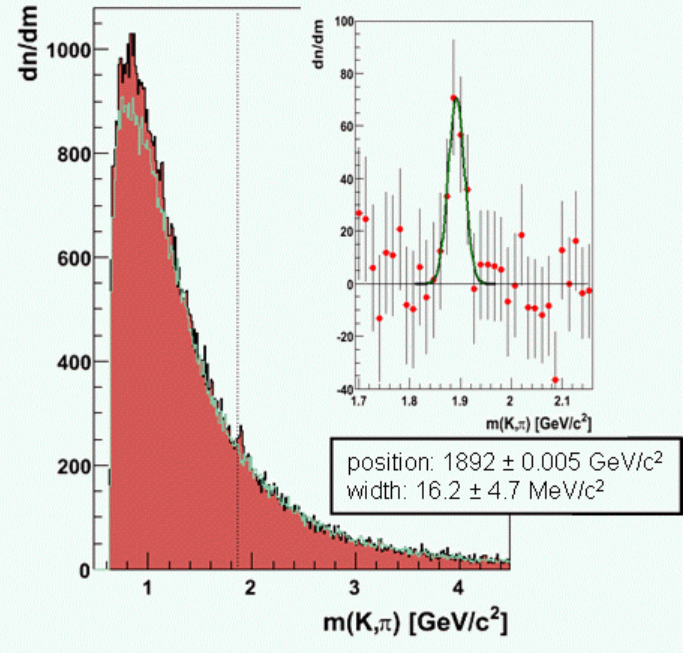

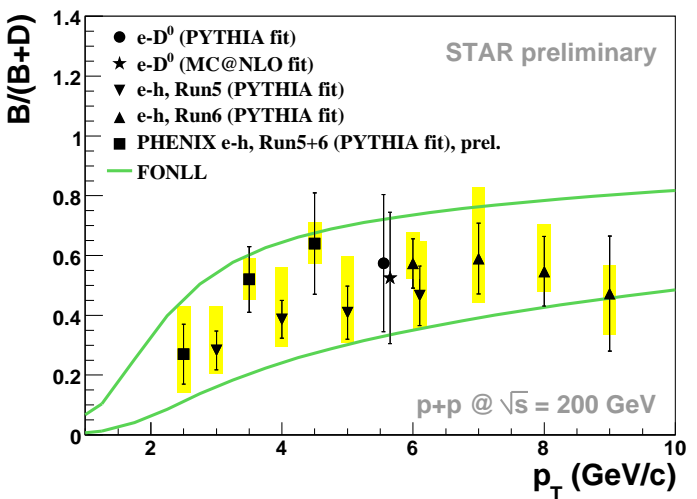

Figure 2. Left: The $\mathrm{K} \pi$ invariant mass in $200 \mathrm{GeV}$ p + p collisions using a non-photonic electron trigger as measured by STAR. The inset shows a background subtracted distribution. Right: $\mathrm{B} /(\mathrm{B}+\mathrm{D})$ ratio as measured by STAR and PHENIX compared to FONLL calculations. 


\section{Dileptons and Direct Photons}

As we explore the properties of the sQGP, we wish to obtain information from early times in the evolution of the collision. Since they do not experience the strong interaction, leptons and photons are ideal messengers of information from deep within the fireball. They can give us vital information on two fronts. The first, via leptonic decays of hadrons (or at least quasi-hadrons), is information on the characteristics of hadrons in the high temperature environment. They will tell us of modifications to the vacuum hadronic states - in particular - whether chiral symmetry is restored. Dileptons and photons can also give information on the early temperature, via the black body radiation spectrum.

Leaving aside the question of whether the sQGP is formed at the SPS or not, we turn to the NA60 experiment which has made a precision measurement of the low mass dimuon spectrum in semi-central In+In collisions [8] (see Fig. 3, left). Note that the atomic weight of In is 115, about half the atomic weight of lead or gold used in previous SPS experiments and RHIC. The model of Rapp and Wambach where the $\rho$ spectral function is modified fits the invariant mass spectrum - the data is consistent with a broadening of the $\rho$ with no mass shift. This is an important result, as hadronic models are essentially an attempt to do many body QCD calculations below $\mathrm{T}_{C}$ and include an approach to the restoration of chiral symmetry as $\mathrm{T}_{C}$ is approached from below (see [9]). Theoretical calculations show that baryons are important for this effect. When baryons are taken out of the calculation, the models are no longer able to explain the data. It then is reasonable to conjecture that the effect seen in NA60 is a result of the baryon density at the SPS. Generally the modification of particle masses and widths are a slow function of the baryon density below the critical temperature. Even at the baryon density of nuclear matter, meson masses and widths are expected to be modified $t$.

In the model, the excess on the high mass side of the $\rho$ is not explained. In order to study this region, the NA60 collaboration divided the data into sub-samples of varying invariant mass, and then fitted the $\mathrm{p}_{T}$ spectra of the sub-sample. The resultant slopes are shown in Fig. 3 (right). They note that for masses below about $800 \mathrm{MeV}$ - i.e. below the $\rho$ - the slope of the $\mathrm{p}_{T}$ spectra rises. This is reminiscent of the mass dependent $\mathrm{p}_{T}$ spectra of hadrons - an indicator of radial flow of a hadronic source i.e. primarily the $\rho$. The radial flow is built up in the later stages of the collision which is dominated by hadrons. Above $\sim 800 \mathrm{MeV}$, the slopes drop, which could be an indicator that the source of at least some of such dileptons are from earlier in the collision, i.e. before the radial flow develops [10]. This might imply that the source is of a partonic nature.

One of the new results shown at this conference by NA60 was an analysis of

the polarization by examining the polar angle of the excess dimuons in the Collins

$\ddagger$ In contrast, as a function of temperature, the modification of hadrons is rather sharp in the region of $\mathrm{T}_{C}$ for low baryon density. It is interesting to note, however, that in many of these models, even in RHIC collisions where the net baryon density is low, broadening of the meson masses is due to baryons - however in this case it is due to the non-equilibrium presence of baryon and their corresponding antibaryons [1]. 
Soper Frame. Within errors the distribution is consistent with an unpolarized source as expected of thermal radiation[12.
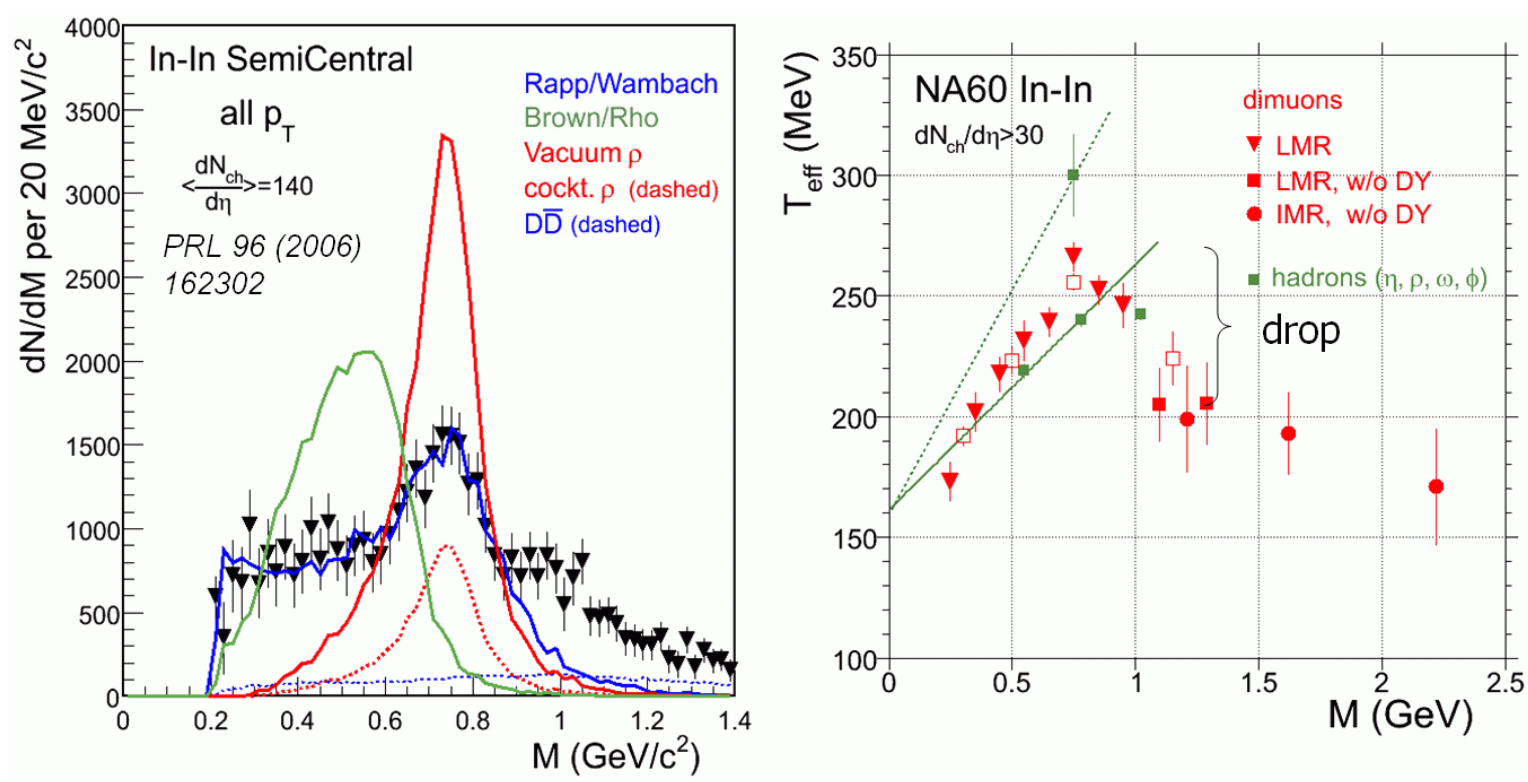

Figure 3. Left: The dimuon invariant mass spectrum in semi-central In+In collisions measured by NA60, as compared to models. Right: Inverse slope parameter for various mass bins.

Now we turn to the RHIC data. In particular, since we have strong evidence for thermalization above the critical temperature, and since there is no doubt that QCD is the governing theory, we must detect thermal radiation, and chiral symmetry must be restored. What we would like to know is, a) a measure of the temperatures involved and b) the mechanism and consequences of the restoration of chiral symmetry. At this conference we have seen two crucial results from the PHENIX collaboration on this subject. The first is the observation of an excess in the dilepton spectrum over what would be predicted from a hadronic cocktail as shown in Fig. 4(left). In the figure, the normalization of the $\mathrm{p}+\mathrm{p}$ and cocktail spectra to the $\mathrm{Au}+\mathrm{Au}$ data is done in the region of the $\pi^{0}$. Fig. 4(right) shows that the $\rho$ broadening model which was successful in explaining the NA60 data no longer works in the region of invariant mass between 150 and $750 \mathrm{MeV}$. So far, no model appears able to explain the data. We then look at the general behavior of the excess as a function of centrality and transverse momentum. Fig. 5 (left) shows the centrality dependence of the excess in three mass regions: the $\pi^{0}$ region where the data is normalized, a low mass region between 0.15 and $0.75 \mathrm{GeV} / \mathrm{c}^{2}$, and a high mass region between 1.2 and $2.8 \mathrm{GeV} / \mathrm{c}^{2}$. In the high mass region, the excess scales with binary collisions, and agrees with a cocktail which includes charm. In the low mass region, the excess increases with centrality. The $\mathrm{p}_{T}$ dependence of the low mass region is shown in Fig. 5(right). The excess is strongest in the lowest $\mathrm{p}_{T}$ bin $(0-0.7 \mathrm{GeV} / \mathrm{c})$ and is reduced at the highest $\mathrm{p}_{T}$. The fact that the excess is strongest in the most central, low $\mathrm{p}_{T}$ collisions is consistent with the signal coming from 
a modified hadron in the fireball. Particles at low $\mathrm{p}_{T}$ from central events, would have presumably been exposed for the longest time to the highest energy density region of the collision. The data is subdivided into bins of invariant mass, and the inverse slopes of the $\mathrm{p}_{T}$ distribution are fit. Since there is clearly a strong $\mathrm{p}_{T}$ dependence which may indicate different sources, the fit is done in a low $\mathrm{p}_{T}$ region of $0<m_{T}<1 \mathrm{GeV} / c^{2}$ and an intermediate $\mathrm{p}_{T}$ region of $1<m_{T}<2 \mathrm{GeV} / c^{2}$. The high $\mathrm{p}_{T}$ region (with large error bars) gives a rising inverse slope consistent with the source being the radial flow of modified hadrons (the $\rho$ ?), albeit as mentioned previously, models including this effect cannot completely describe the excess and alternate explanations may be in order. The low $\mathrm{p}_{T}$ region fits to a slope of about $120 \mathrm{MeV} / \mathrm{c}^{2}$ which seems low to be from a partonic source in the sQGP since it is below any of the standard values of $\mathrm{T}_{C}$ from the lattice [13.
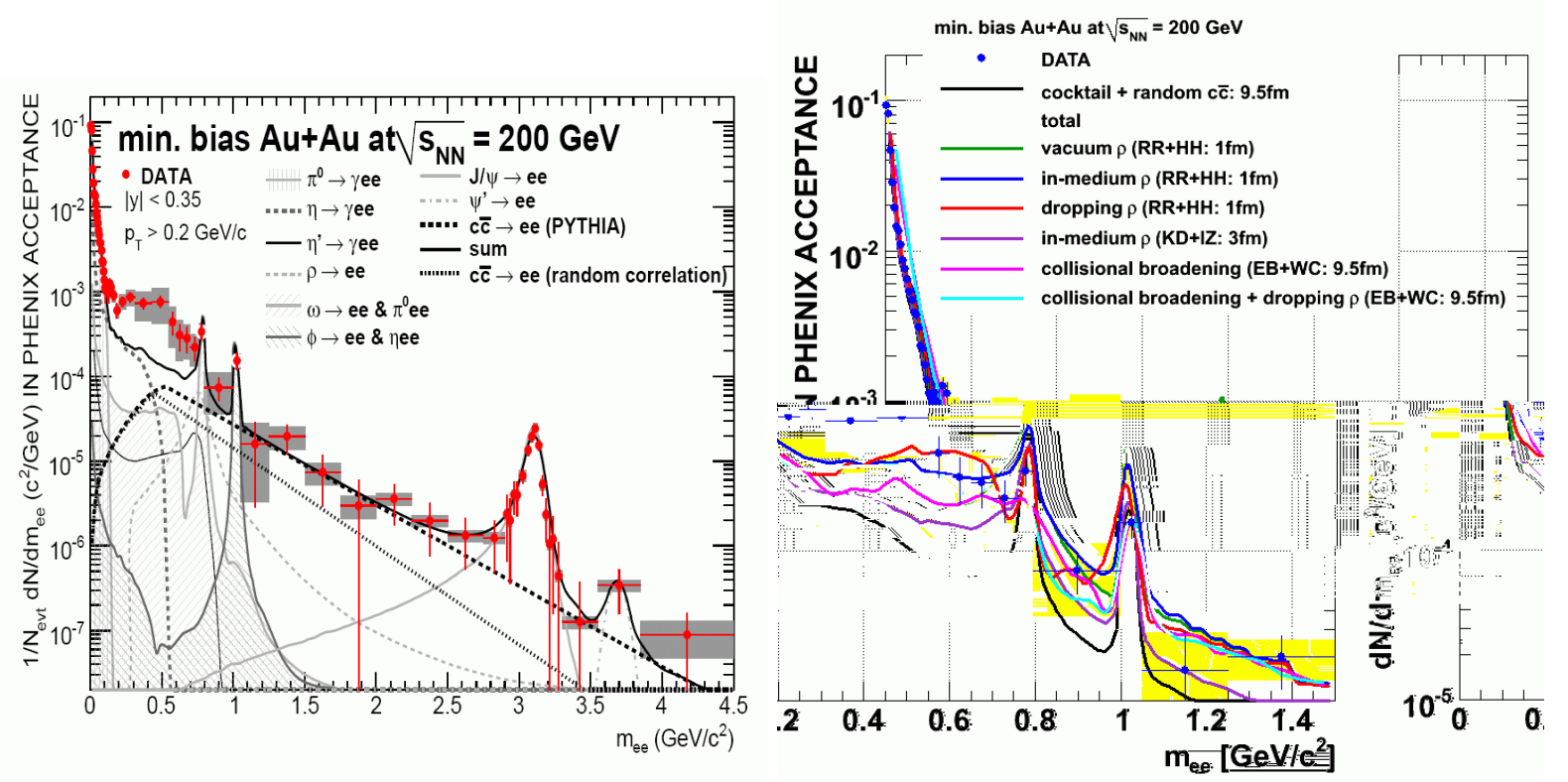

Figure 4. Left: The dielectron mass spectrum as compared to $\mathrm{p}+\mathrm{p}$ and hadronic cocktail normalized at $\mathrm{m}_{e e}<20 \mathrm{MeV} / \mathrm{c}^{2}$ as measured by PHENIX in $\mathrm{p}+\mathrm{p}$ and $\mathrm{Au}+\mathrm{Au}$ at $200 \mathrm{GeV}$. Right: The dielectron invariant mass spectrum compared to models.

A second new result is the $\mathrm{p}+\mathrm{p}$ confirmation to the background of the excess in virtual direct photons as detected through the dilepton channel shown at QM2005. Photon measurements are very difficult due to the large background from photons from $\pi^{0} \mathrm{~s}$. A way to overcome this is to look at virtual photons which internally convert into dielectrons. If one cuts on an invariant mass above the $\pi^{0}$ mass, this effectively suppresses the contribution from the $\pi^{0}$ decay. Fig. [6(left two panels) shows the dilepton invariant mass as compared to the cocktail. The cocktail and data are normalized in the region $\mathrm{m}_{e e}<30 \mathrm{MeV}$. For $\mathrm{p}+\mathrm{p}$ collisions the cocktail and data match well, aside from a small excess at high $\mathrm{p}_{T}$ (see Fig. 6, left). In Au+Au collisions one sees an excess when one looks above the $\pi^{0}$ mass. The data is then fit to a combination of cocktail and a spectrum assumed to come from the internal conversion of direct photons. The factor 

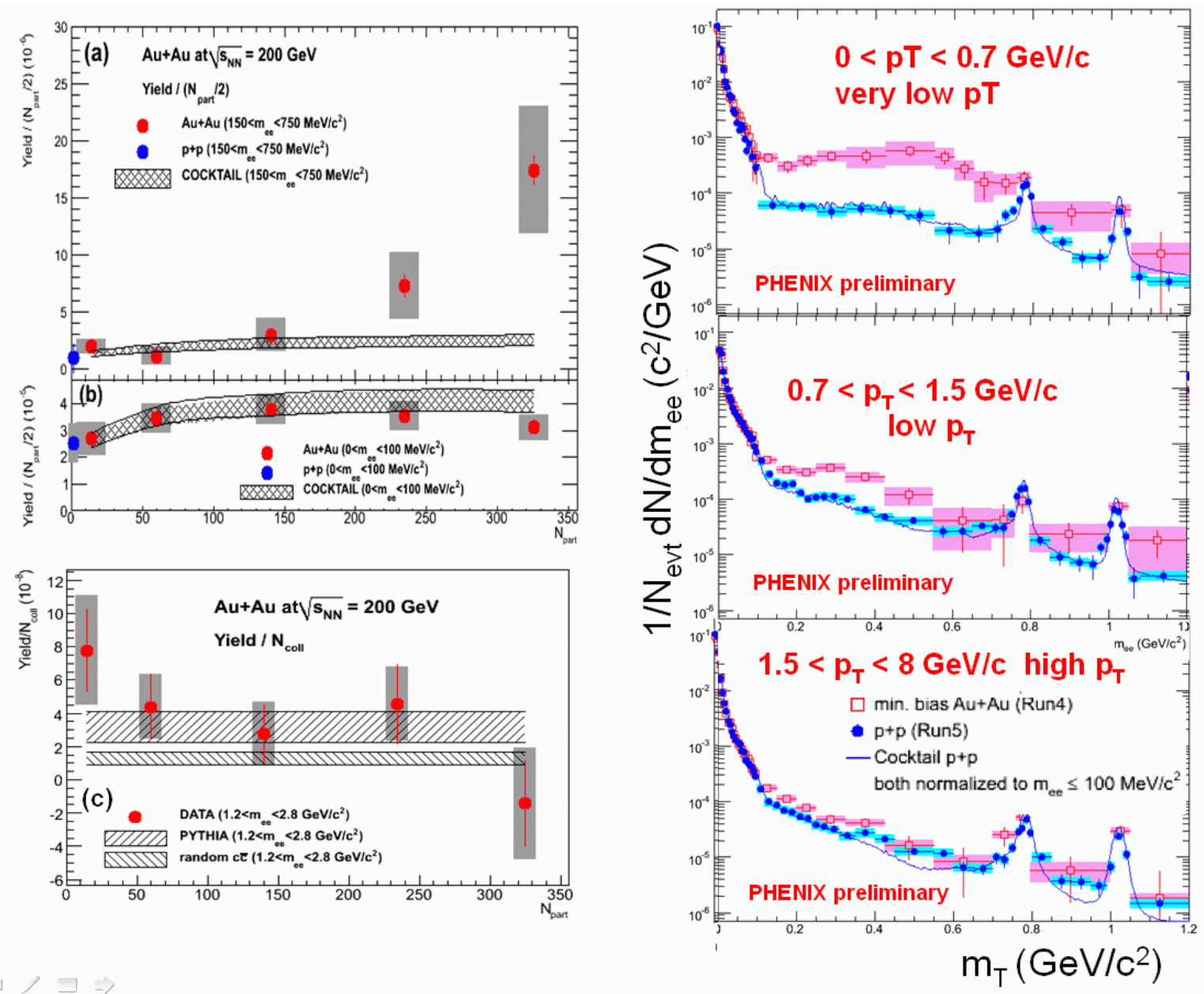

Figure 5. Left: Centrality dependence of dielectron excess in various mass regions as measured by PHENIX in $200 \mathrm{GeV} \mathrm{Au}+\mathrm{Au}$ collisions. The top panel is for the low mass region and is normalized to $\mathrm{N}_{\text {part }}$. The center panel is for the $\pi^{0}$ mass region where the data is normalized to the cocktail. The bottom panel shows the high mass region normalized to the number of binary collisions. Right: The invariant mass spectrum in 3 regions of $\mathrm{p}_{T}$.

$r$ which governs the strength of the internal conversion component is free to vary. If one compares the measured value of $r$ to that expected from a NLO pQCD calculation the $\mathrm{p}+\mathrm{p}$ data nicely match, while the $\mathrm{Au}+\mathrm{Au}$ data show a large excess (see [14]). The fraction of direct photons is then multiplied by the inclusive photon yield and a cross section is produced (see Fig. 6, right). A fit to the spectrum from the $20 \%$ most central collisions to an exponential plus a $\mathrm{T}_{A A}$-scaled $\mathrm{p}+\mathrm{p}$ fit function, yields an inverse slope of $221 \pm 23 \pm 18 \mathrm{MeV}$. Assuming that the direct photons are of a thermal origin, the extracted inverse slope can be related to an initial temperature $\mathrm{T}_{\text {init }}$. Hydrodynamic models which include the evolution of the system yield between 300 and $600 \mathrm{MeV}$ for the initial temperature, where $\mathrm{T}_{\text {init }}$ is strongly dependent on the initial thermalization time; as one might expect, later times yield lower temperatures[15]. 

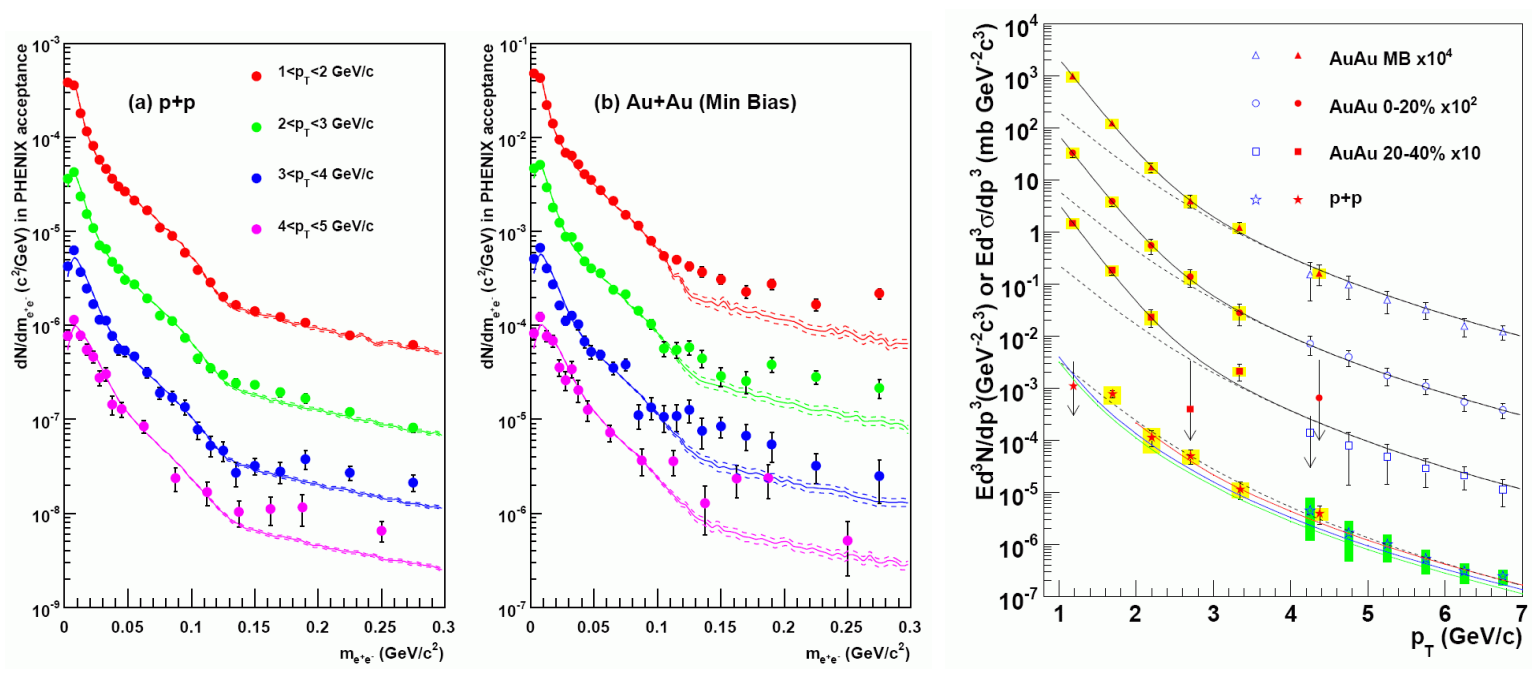

Figure 6. Left 2 panels: the dielectron invariant mass spectrum in $200 \mathrm{GeV} \mathrm{p}+\mathrm{p}$ and $\mathrm{Au}+\mathrm{Au}$ collisions as compared to a hadronic cocktail. Right panel: Invariant cross section $(\mathrm{p}+\mathrm{p})$ and invariant yield $(\mathrm{Au}+\mathrm{Au})$ of direct photons as a function of $\mathrm{p}_{T}$. The three curves on the $\mathrm{p}+\mathrm{p}$ data represent NLO pQCD calculations, and the dashed curves show a modified power-law fit to the $\mathrm{p}+\mathrm{p}$ data, scaled by $\mathrm{T}_{A A}$. The black curves are exponential plus the $\mathrm{T}_{A A}$ scaled $\mathrm{p}+\mathrm{p}$ fit.

\section{The $\mathbf{J} / \psi$}

The $\mathrm{J} / \psi$ data at RHIC presents a conundrum. Is $\mathrm{J} / \psi$ suppression a sure sign of deconfinement? At RHIC, the possibility of production through coalescence is an important consideration, as is the initial state of the nucleus - the Colored Glass Condensate, or shadowing, absorbed under the general heading of "cold nuclear effects". While this is an experimental review, one of the encouraging new results shown at this meeting for experimentalists was theoretical. For the past few years potential models which are used to make predictions for heavy ion collisions and lattice results have not agreed. At this conference agreement was shown between a potential model and lattice correlators for all states [16, 17]. This goes a long way to putting the suppression via deconfinement models on stronger footing. The "melting temperatures" of the various charmonium have changed from previous values (see Fig. 17). The possibility of coalescence and initial state effects are still present. However, it is clear that charmonium can tell us something about deconfinement, if we can disentangle deconfinement from other effects. This will require both improvements to dynamic model calculations including coalescence, and good experimental data sets in proton-nucleus or deuteronnucleus collisions to understand cold nuclear matter effects.

Data from PHENIX including $\mathrm{Cu}-\mathrm{Cu}$ results, and a first look at a larger sample of $\mathrm{Au}+\mathrm{Au}$ data are consistent with previous results (see Fig. 8, left). Naively one would think the energy density is lower in the forward rapidity region, therefore the effect of deconfinement would be weaker. Just the opposite is seen. Suppression at forward rapidity is stronger than at midrapidity! One then turns to the $\mathrm{d}+\mathrm{Au}$ data to 
understand the effect of the initial state on the suppression. Fig. 8 (right two panels) shows the projection of the cold nuclear matter effect, taken from $\mathrm{d}+\mathrm{Au}$ measurements. A data driven technique is used in which it is assumed that a single modification factor which is a function of the radial position in the nucleus parametrizes all cold nuclear matter effects [18]. Unfortunately the quality of the $\mathrm{d}+\mathrm{Au}$ data is too poor to give a conclusive answer. A recently obtained data set should give PHENIX a sample 30 times as large which should go a long way to pinning down the cold nuclear matter effects. Future measurements of the $\psi^{\prime}, \chi_{C}$, and $\Upsilon$ states, together with open charm and bottom measurements in $\mathrm{p}+\mathrm{p}, \mathrm{d}+\mathrm{Au}$, and $\mathrm{Au}+\mathrm{Au}$ collisions should provide enough information to tease apart the the different phenomena - i.e. cold nuclear effects, coalescence, and deconfinement.

One of the interesting predictions coming into this conference from AdS/CFT is that the suppression of the $\mathrm{J} / \psi$ would increase with $\mathrm{p}_{T}[19]$. A two component model which includes dissociation and regeneration [20] predicts a flat $\mathrm{p}_{T}$ with coalescence dominating at low $\mathrm{p}_{T}$ and dissociation at high $\mathrm{p}_{T}$. Early predictions based on a simple potential model[21] predict less suppression as $\mathrm{p}_{T}$ is increased. STAR has made a first attempt to look at the very high $\mathrm{p}_{T}$ dependence of the $\mathrm{J} / \psi$ via its decay to dielectrons identified in a combination of the TPC and EMC and triggered by the EMC (Fig. 9). The figure also shows the PHENIX data. At the moment the results are inconclusive, but it will be interesting to see the dependence of the $\mathrm{J} / \psi$ on transverse momentum once the two collaborations get higher statistics data. This depends critically on the available running time at RHIC to obtain good statistics at high $\mathrm{p}_{T}$.

\begin{tabular}{|lcccccc|}
\hline state & $\chi_{c}$ & $\psi^{\prime}$ & $J / \psi$ & $\Upsilon^{\prime}$ & $\chi_{b}$ & $\Upsilon$ \\
\hline$T_{\text {dis }}$ & $\leq T_{c}$ & $\leq T_{c}$ & $1.2 T_{c}$ & $1.2 T_{c}$ & $1.3 T_{c}$ & $2 T_{c}$ \\
\hline
\end{tabular}

Figure 7. Quarkonium dissociation temperatures from [17] .

\section{Conclusion}

In closing there were two main results in the realm of leptons, photons and heavy quarks that we should take away from this conference:

- Bottom quarks flow and are suppressed at high $\mathrm{p}_{T}$ in heavy ion collisions.

- We are now beginning to see a signal for enhanced photon and dilepton production in heavy ion collisions.

There are clearly questions to be answered in the coming years:

- What are the sources of the excess dileptons/photons?

- Are they telling us the temperature? What is it? 


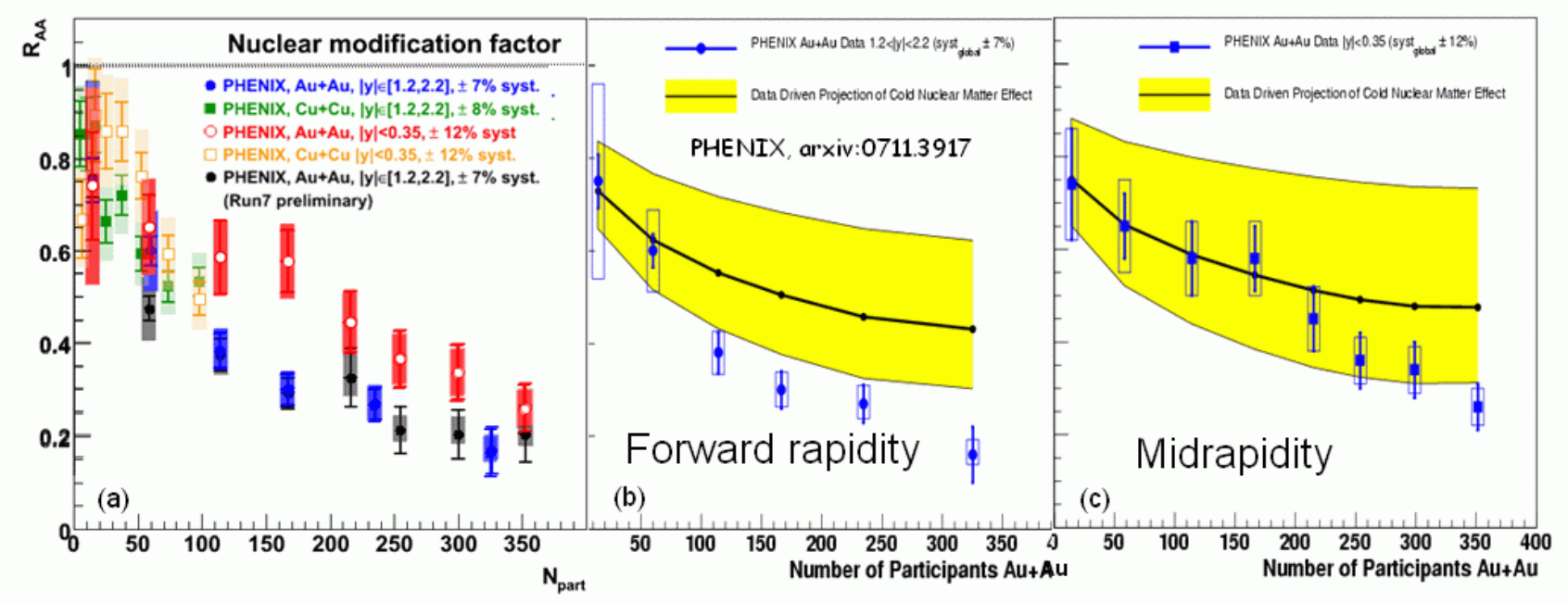

Figure 8. Left panel: $\mathrm{R}_{A A}$ for the $\mathrm{J} / \psi$ as measured by PHENIX. Note that the forward rapidity region shows more suppression than the mid-rapidity region. Right two panels: The forward and mid rapidity data together with a projection of the cold nuclear matter effect, using PHENIX $\mathrm{d}+\mathrm{Au}$ data.

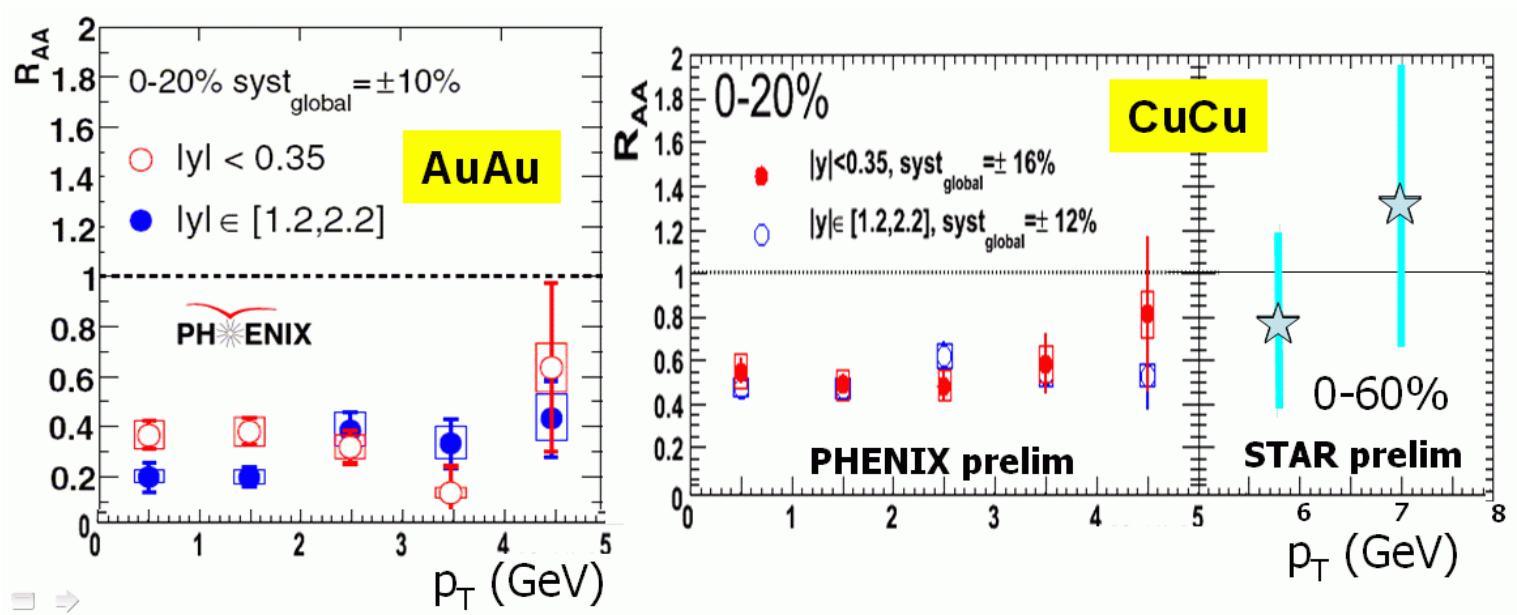

Figure 9. The $\mathrm{p}_{T}$ dependence of the $\mathrm{J} / \psi \mathrm{R}_{A A}$ as measured by PHENIX and STAR.

- How do the heavy quarks manage to become part of the bulk? How strong is the "s" in sQGP. What is the viscosity?

- What is the mechanism of the rapid thermalization, and the thermalization or partial thermalization of heavy quarks?

- Does the $\mathrm{J} / \psi$ show anomalous suppression due to deconfinement?

It is an exciting time for our field. We now have many powerful tools at our disposale.g. the flow of the charmonium to test models of coalescence and deconfinement, correlations measurements between photons and jets, or charm quarks and jets, precision measurements of the dilepton and photon spectra. In addition the LHC will be coming on in the next few years bringing a host of new information from higher energies. I 
would like to close with one important fact. All of the heavy ion experiments both at RHIC and at the LHC depend and will depend on adequate running time, and support for the detectors and analysis. The possibilities ahead of us are tremendous, but we must ensure that support for the field does not waver, else we will leave many of these questions unanswered.

\section{References}

[1] U. W. Heinz, AIP Conf. Proc. 739, 163 (2005) arXiv:nucl-th/0407067.

[2] M. Strickland, J. Phys. G 34, S429 (2007) arXiv:hep-ph/0701238.

[3] Y. L. Dokshitzer and D. E. Kharzeev, Phys. Lett. B 519, 199 (2001) arXiv:hep-ph/0106202.

[4] A. Adare et al. [PHENIX Collaboration], Phys. Rev. Lett. 98, 172301 (2007) arXiv:nucl-ex/0611018.

[5] H. van Hees and R. Rapp, Phys. Rev. C 71, 034907 (2005) arXiv:nucl-th/0412015.

[6] G. D. Moore and D. Teaney, Phys. Rev. C 71, 064904 (2005) arXiv:hep-ph/0412346.

[7] G. Policastro, D. T. Son and A. O. Starinets, Phys. Rev. Lett. 87, 081601 (2001) arXiv:hep-th/0104066.

[8] R. Arnaldi et al. [NA60 Collaboration], Phys. Rev. Lett. 96, 162302 (2006) arXiv:nucl-ex/0605007.

[9] R. Rapp, Nucl. Phys. A 782, 275 (2007) arXiv:nucl-th/0608022.

[10] R. Arnaldi et al. [NA60 Collaboration], Phys. Rev. Lett. 100, 022302 (2008) arXiv:0711.1816 [nucl-ex]].

[11] R. Rapp, arXiv:nucl-th/0204003.

[12] See S. Damjanovic, these proceedings.

[13] See A. Toia, these proceedings.

[14] See T. Dahms, these proceedings.

[15] A. Adare et al. [PHENIX Collaboration], Submitted to Physical Review Letters, arXiv:0804.4168v1 [nucl-ex].

[16] A. Mocsy and P. Petreczky, Phys. Rev. D 77, 014501 (2008) [arXiv:0705.2559 [hep-ph]].

[17] A. Mocsy and P. Petreczky, Phys. Rev. Lett. 99, 211602 (2007) arXiv:0706.2183 [hep-ph]].

[18] A. Adare et al. [PHENIX Collaboration], Phys. Rev. C 77, 024912 (2008) arXiv:0711.3917 [nuclex]].

[19] H. Liu, K. Rajagopal and U. A. Wiedemann, Phys. Rev. Lett. 98, 182301 (2007) arXiv:hep-ph/0607062.

[20] X. Zhao and R. Rapp, arXiv:0712.2407 [hep-ph].

[21] J. P. Blaizot and J. Y. Ollitrault, Phys. Lett. B 199 (1987) 499. 